Pathogenic ELN gene mutations (ELN, MIM\#130160) cause AD Supravalvular Aortic Stenosis (SVAS) a congenital narrowing of the ascending aorta, and Cutis Laxa (CL) characterised by inelastic, loose-hanging skin. Variable phenotype and penetrance is apparent. Pathogenic ELN variants result in loss of function and include frameshift (most common), nonsense, splice site and missense variants. The well characterised contiguous gene deletion syndrome, Williams-Beuren syndrome includes SVAS and encompasses at least $114 \mathrm{~kb}$ on $7 \mathrm{q} 11.23$ including the ELN gene; however, there are only 5 case reports of CNVs within ELN (single or multiple exons).

Bristol Genetics Laboratory provides a UKGTN approved service for ELN gene sequencing (33 coding exons). In three years, $52 \mathrm{UK}$ and foreign patients with SVAS, CL or features such as pulmonary artery stenosis and aortic dilation have been tested. 18/52 (34\%) patients were heterozygous for a likely pathogenic variant including frameshift (6), nonsense (4), splice (4), and missense (4). 12 of these cases were novel variants, 5 are supported by segregation analysis and 1 is sporadic. The remaining novel variants are classed as possibly pathogenic as they are phenotypically compatible.

$12 / 35$ patients negative on sequencing have so far been screened for CNVs by MLPA (MRC Holland) covering the Williams-Beuren syndrome region, including 10 exons of the ELN gene (1, 3, 4, 6, 9, 16, 20, 26, 27 and 33) and in addition a bespoke MLPA assay including probes for exons 28 to 30, 32 and 3'UTR.

4/12 (33\%) patients have a heterozygous deletion within the ELNgene. A mother and daughter with pulmonary stenosis and an extended family history have a deletion spanning exons 30 to 33. This deletion was also identified in another patient with SVAS and arteriopathy. A deletion of the 5' end of the gene, involving at least exon 1 (but not exon 3) was identified in an infant with SVAS and pulmonary branch stenosis, and a deletion involving the entire coding region of the ELN gene and at least the first two exons of the adjacent 3' gene LIMK1 was detected in a neonate who died at 2 months with SVAS, pulmonary stenosis and mild hypoplasia with PDA. The deletion was detected in this patient's father who consequentially was found to have an aortic regurgitation and in a subsequent pregnancy of this family which was lost at 31 weeks with pulmonary stenosis and significant aortic stenosis

MLPA analysis has enhanced the clinical utility of this service giving an increased diagnostic yield in patients with SVAS and CL and related presentations.

\section{GLYCOMIMETICS; A NOVEL CLASS OF DRUGS TO PROTECT AGAINST FREE FATTY ACID-INDUCED ENDOTHELIAL DYSFUNCTION}

${ }^{1}$ Fiona L Wilkinson*, ${ }^{2}$ Ayman Mahmoud, ${ }^{1}$ Alan M Jones, ${ }^{3}$ James Wilkinson, ${ }^{4}$ Miguel Romero, ${ }^{4}$ Juan Duarte, ${ }^{1} \mathrm{M}$ Yvonne Alexander. ${ }^{1}$ Manchester Metropolitan University; ${ }^{2}$ Beni-Suef University; ${ }^{3}$ University of Salford; ${ }^{4}$ University of Granada; * Presenting Author

\subsection{6/heartjnl-2016-309890.204}

Background Endothelial dysfunction is a key player in cardiovascular disease (CVD) complications and novel drugs are required to treat this pathological process. Glycosaminoglycans (GAGs) are key molecules that regulate signalling in many biological processes and drugs that mimic their structure could be a novel source of therapeutics to target specific CVD pathways.

Purpose We have synthesised a set of four glycomimetic compounds and our objective was to determine whether they could activate protective pathways in endothelial cells subjected to fatty acid-induced endothelial dysfunction.

Methods Glycomimetics, C1-C4, were synthesised by the stepwise transformation of 2,5-dihydroxybenzoic acid to a range of 2,5-substituted benzoic acid derivatives, incorporating the key sulphate groups to mimic heparan sulphate. Human Umbilical Vein Endothelial Cells (HUVECs) were treated with glycomimetics $(1 \hat{A} \mu \mathrm{M})$ in the presence or absence of the free fatty acid, palmitate. DAF-2 and $\mathrm{H}_{2}$ DCF-DA assays were used to determine $\mathrm{NO}$ and reactive oxygen species (ROS) production, respectively. Lipid peroxidation colorimetric and antioxidant enzyme activity ssays were also carried out. RT-PCR and western blotting were utilised to measure Akt, eNOS, Nrf-2, NQO-1 and HO-1 expression. Endothelial function was determined ex vivo using acetylcholine-induced endotheliumdependent relaxation in mouse thoracic aortic rings by wire myography.

Results All four glycomimetics protected against palmitateinduced oxidative stress and enhanced $\mathrm{NO}$ production in vitro via upregulation of Akt/eNOS signalling, activation of the Nrf2/ARE pathway and down-regulation of ROS-induced lipid peroxidation. Under palmitate-induced oxidative stress, ex vivo endothelium-dependent relaxation was significantly enhanced by all four glycomimetics. Furthermore, the glycomimetics did not induce HUVEC activation, as determined by lack of ICAM-1 protein.

Conclusion We have developed a new set of small molecule glycomimetics that do not activate ECs and protect against free fatty acid-induced endothelial dysfunction both in vitro and ex vivo. Future work will focus on developing the glycomimetics into drug-like therapies that target endothelial damage.

\section{INFLUENCE OF NOX NADPH OXIDASES ON HUMAN PARTIAL INDUCED PLURIPOTENT STEM CELL-DERIVED ENDOTHELIAL CELLS}

Arya Moez*, Karla O’Neill, Andriana Margariti, David Grieve. Queen's University Belfast; *Presenting Author

\subsection{6/heartjnl-2016-309890.205}

Background Human induced pluripotent stem (iPS) cell-derived endothelial cells (ECs) hold clear potential for therapeutic angiogenesis as a novel strategy for ischaemic disease. Recently, our group has developed a novel method for direct reprogramming of partial iPS (PiPS) cells, which unlike iPS cells, are generated before pluripotency so do not form tumours. Importantly, PiPS cells may be differentiated into ECs with characteristic morphology and pro-angiogenic actions, which in vitro and in vivo studies have demonstrated are comparable to mature ECs with regard to their capability of forming vascular-like tubes and re-endothelialisation of ischaemic tissue. It is well established that oxidative stress and reactive oxygen species (ROS), which are characteristic features of ischaemic disease, are important regulators of both endothelial and stem cell biology, with recent evidence suggesting a key role for NADPH oxidases. Notably, we have previously identified a key role for the Nox4 isoform in regulating 
angiogenesis. The aim of the present study was therefore to investigate the specific influence of Nox NADPH oxidases on PiPS-derived ECs.

Methods Human neonatal lung fibroblasts were subjected to direct reprogramming by viral transduction with the transcription factors, OCT4, SOX2, Klf4 and c-Myc, for 4 days to generate PiPS cells, which were then seeded on mouse collagen IV in EGM-2 media for a further 6 days to induce EC differentiation.

Results PiPs cells were defined by induced expression of KDR and CD34, whilst PiPS-ECs were characterised by expression of endothelial markers, CD144 and CD31. Expression of NADPH oxidase isoforms, Nox1, Nox2 and Nox4 (as assessed by real-time RT-PCR) was progressively induced during reprogramming and EC differentiation (e.g. Nox4 mRNA: fibroblasts, $2.1 \pm 0.2$; PiPS, $36 \pm 4$; PiPS-ECs, $133 \pm 29$ arbitrary units; $\mathrm{n}=3, \mathrm{P}<0.001$ ), with comparable levels observed in PiPS-ECs (Nox1 mRNA, $126 \pm 20$; Nox2 mRNA, $82 \pm 20$; Nox4 mRNA, $133 \pm 29$ arbitrary units; $n$ $=3, \mathrm{P}=\mathrm{NS}$ ). Notably, mRNA expression of Nox4, which has been previously linked with angiogenesis, was markedly increased during hypoxia (1\% oxygen) in PiPS-ECs compared with the other two isoforms (Nox1, $2.89 \pm 0.26$; Nox2, 1.52 \pm 0.06 ; Nox $4,8.04 \pm 0.76$ arbitrary units; $\mathrm{n}=3, \mathrm{P}<$ 0.001).

Conclusions These data suggest that NADPH oxidases, particularly Nox4, may be important in mediating angiogenic function in PiPS-ECs. Future work will investigate whether genetic modification of Nox4 can influence reprogramming and differentiation of PiPS-ECs and consequently their ability to promote angiogenesis in response to experimental ischemia.

\section{B CELL-SPECIFIC FC GAMMA RECEPTOR IIB OVER- EXPRESSION ENHANCES ATHEROSCLEROSIS BY INHIBITING B1 CELLS}

Andrew Sage*, Deirdre Murphy, Leanne Masters, Lauren Kitt, Reem Assil, Ziad Mallat. University of Cambridge; *Presenting Author

\subsection{6/heartjnl-2016-309890.206}

Antibodies binding oxidised epitopes on apoptotic cells and oxidised forms of LDL form an important protective barrier slowing the development of atherosclerosis. However, not all $\mathrm{B}$ cell functions or subsets are protective since adaptive B2 cell depletion is atheroprotective and autoimmunity is associated with accelerated atherosclerosis. The inhibitory IgG receptor $\mathrm{FcI}^{3} \mathrm{RIIb}$ is the only IgG receptor found on $\mathrm{B}$ cells and also counteracts pro-inflammatory signalling from activating $\mathrm{FcI}^{3}$ receptors in macrophages and dendritic cells. Studies on $\mathrm{FcI}^{3} \mathrm{RIIb}$ knockout mice demonstrate significant regulation of atherosclerosis but contrasting effects depending on the genetic background, suggesting the need for alternative approaches. We have analysed the effects of B cell-specific over-expression of $\mathrm{Fc}^{3} \mathrm{RIIb}$ using a previously characterised transgenic mouse strain. $\mathrm{FCl}^{3} \mathrm{RIIb}$ over-expression was confirmed to be specific for $\mathrm{B}$ cells and was equal between genders. There was only minor impacts on mature $\mathrm{B}$ cell levels, but $\mathrm{Fc}^{3} \mathrm{RIIb}$ overexpression significantly attenuated plasma cell levels and serum antibody titres. Female, but not male, Fcîl ${ }^{3} \mathrm{RIIb}-\mathrm{B}$ cell transgenic mice on the $\mathrm{ApoE}^{-/-}$background develop significantly enhanced atherosclerosis after 6 weeks high fat diet compared to non-transgenic littermates. Females but not males had significantly decreased oxidised epitope-specific IgM levels and $\mathrm{B} 1 \mathrm{a}$ cells in spleen and peritoneal lavage. In vitro, B1 cells from female $\mathrm{ApoE}^{-/-}$mice were more susceptible to $\mathrm{Fc}^{3} \mathrm{RIIb}-$ induced apoptosis. Overall, our study highlights a potential difference in B1 cell biology between males and females, and supports the investigation of gender specific effects of autoimmune-linked $\mathrm{Fc}^{3} \mathrm{RIIb}$ polymorphisms on human cardiovascular disease.

\section{THE INTERPLAY BETWEEN THE RENIN ANGIOTENSIN SYSTEM AND PACING POSTCONDITIONING INDUCED CARDIAC PROTECTION}

Fawzi Babiker*, Aishah Al-Jarallah, Shaji Joseph. Kuwait University; *Presenting Author

\subsection{6/heartjnl-2016-309890.207}

Background Accumulating evidence suggests a cardioprotective role of pacing postconditioning (PPC). The interaction between RAS and PPC induced cardiac protection is however not clearly understood. The role of angiotensin converting enzyme (ACE), Angtiosin II (Ang II) and angiotensin receptor 1 (AT1) remains to be identified.

Objective The objective of this study was therefore to investigate the role of ACE-Ang II-AT1 axes of RAS in the protective effects of PPC.

Methods The role of RAS was tested by infusion of Ang II, chymostatin (inhibitor of locally produced Ang II), ACE blocker (captopril) and AT1 antagonist (irbesartan). Hemodynamics data was computed digitally and infarct size was determined using 2,3,5-Triphenyltetrazolium chloride (TTC) staining and by measuring creatine kinase $(\mathrm{CK})$ and lactate dehydrogenase (LDH) levels.

Results In comparison to hearts subjected to $\mathrm{I} / \mathrm{R}$ injury or untreated control hearts, PPC significantly $(\mathrm{P}<0.001)$ improved cardiac hemodynamics and reduced infarct size and cardiac enzymes. Systemic infusions of Ang II did not affect I/ $\mathrm{R}$ injury or PPC mediated protection. Nonetheless inhibition of endogenously synthesised Ang II protected against I/R induced cardiac damage and did not block or augment the protective effects of PPC. The administration of AT1 antagonist did not alleviate $\mathrm{I} / \mathrm{R}$ induced damage. Interestingly it abrogated PPC induced cardiac protection. Finally, PPC induced protection and blockade of locally produced Ang II involved enhanced activation of ERK1/2 and Akt.

Conclusions This study demonstrate a novel interaction between PPC mediated cardiac protection and ACE-Ang IIAT1 axes of the RAS in which locally produced Ang II appears to play a significant role in PPC mediated protection while systemically produced Ang II appears to be dispensable. Moreover this interactions appears to involve alterations in the activation state of downstream kinases including ERK1/2 and Akt.

Acknowledgement This study is supported by grant number MY 02/10 from Research Administration, Kuwait University, Kuwait. 